\section{Reprodutibilidade e validade do questionário de freqüência de consumo alimentar utilizado em estudo caso-controle de câncer oral}

\section{Reproducibility and validity of a food frequency questionnaire used in a case-control study of oral cancer}

Hellen Chrystine Zanetti Matarazzo'

Dirce Maria Lobo Marchioni ${ }^{2}$

Rejane Augusta de Oliveira Figueiredo'

Betzabeth Slater ${ }^{2}$

José Eluf Neto $^{3}$

Victor Wünsch Filho'

'Departamento de Epidemiologia da Faculdade de Saúde Pública, USP, São Paulo

2Departamento de Nutrição da Faculdade de Saúde Pública, USP, São Paulo ${ }^{3}$ Departamento de Medicina Preventiva da Faculdade de Medicina, USP, São Paulo

Projeto com financiamento da Comissão Européia IC 18-CT97-0222, FAPESP 2001/01768-2 e CNPq 109122-2002-0.

Correspondência: Hellen Chrystine Zanetti Matarazzo. Departamento de Epidemiologia, Faculdade de Saúde Pública, USP. Av. Dr. Arnaldo 715, Cerqueira César, CEP 01246-904, São Paulo, SP, Brasil. Email: hematarazzo@yahoo.com.br

\section{Resumo}

O objetivo deste trabalho foi verificar a reprodutibilidade e validade do questionário de freqüência de consumo alimentar utilizado no Estudo Latino-Americano sobre Câncer Oral e de Laringe. Uma amostra de 35 indivíduos, considerados controle no Estudo Latino-Americano, foi entrevistada por telefone. Foi reaplicado o questionário de freqüência idêntico ao do Estudo Latino-Americano e aplicado um recordatório de 24 horas. A reprodutibilidade foi avaliada pela comparação do consumo alimentar obtido nos dois questionários de freqüência, e a validade pela comparação do consumo obtido no recordatório de 24 horas e no segundo questionário de freqüência. Na comparação entre os questionários foi utilizado o teste de Wilcoxon para medir as diferenças no consumo, o coeficiente de Spearman para avaliar as correlações, a correlação intraclasse para estimar a variação intrapessoal, e o teste Kappa para avaliar a concordância no consumo. Na análise da reprodutibilidade ocorreram diferenças estatisticamente significantes para o consumo de massas e leguminosas. Na análise da validade ocorreram diferenças estatisticamente significantes para manteiga, tubérculos, frango, hortaliças, leguminosas e frutas, sendo que, em geral, o questionário de freqüência superestimou o consumo destes grupos de alimentos. A concordância de classificação por tercis variou de $40 \%$ a $80 \%$ entre os dois questionários de freqüência, e de $31 \%$ a $74 \%$ entre o recordatório e o segundo questionário de freqüência. Os resultados indicam que o questionário de freqüência utilizado no Estudo LatinoAmericano tem boa reprodutibilidade e razoável validade para estimar o consumo de alimentos.

Palavras-chave: Questionário de freqüência de consumo alimentar. Reprodutibilidade. Validade. Estudo caso-controle. Dieta e câncer. 


\section{Abstract}

We examined the reproducibility and the validity of the food frequency questionnaire used in the Latin American Study of Oral and Larynx Cancer. Telephone interviews were carried out with a sample of 35 participants who were controls in that study. The original food frequency questionnaire and a 24-hour recall were applied. The reproducibility was evaluated by the comparison of food consumption described in both food frequency questionnaires, and the validity by the comparison of food consumption in the 24-hour recall and the second food frequency questionnaire. In order to compare questionnaires, we used the Wilcoxon test to measure food consumption differences, the Spearman coefficient to analyze food consumption correlations, the intraclass correlation to estimate intrapersonal variability, and the Kappa test to analyze food consumption agreement. Statistically significant differences in consumption of pasta and vegetables were observed in the reproducibility analysis. The validity analysis showed statistically significant differences for butter, tubercles, chicken meat, vegetables, leguminous and fruits; in general, the food frequency questionnaire over-estimated the consumption of these food groups. Agreement of individuals' classification in tertiles of food consumption ranged from $40 \%$ to $80 \%$ between the two food frequency questionnaires, and from $31 \%$ to $74 \%$ between the 24 -hour recall and the second food frequency questionnaire. These results revealed that the food frequency questionnaire used in the Latin American Study has good reproducibility and reasonable validity for food consumption.

Keywords: Food frequency questionnaire. Reproducibility. Validity. Case-control study. Diet and cancer.

\section{Introdução}

No Brasil, a incidência de câncer evidencia-se à medida que ocorre o envelhecimento da população, resultado do processo de desenvolvimento econômico e social. Atualmente, o câncer constitui a terceira principal causa de morte no país. Na faixa etária acima de 40 anos de idade é a segunda principal causa de morte, sendo até $30 \%$ desses cânceres diretamente relacionados aos hábitos alimentares ${ }^{1-3}$.

Nos estudos epidemiológicos que investigam associação entre dieta e doenças crônicas não-transmissíveis, inclusive o câncer, em geral é utilizado o questionário de freqüência de consumo alimentar (QFCA) para registrar a dieta dos indivíduos'. Esse instrumento estima o consumo habitual individual durante um determinado período de tempo. Tem como vantagens o baixo custo, a rápida aplicação e a fácil utilização $0^{4,5}$.

Entretanto, os instrumentos que registram o consumo de alimentos, inclusive o questionário de freqüência, são imperfeitos. São étnica e culturalmente sensíveis, e podem estar sujeitos a dois tipos de erros que afetam a qualidade dos dados e, conseqüentemente, os resultados do estudo: erro aleatório e/ou erro sistemático ${ }^{6,7}$. O erro aleatório é a variação da medida que não está relacionada com nenhuma outra medida e que se considera devida ao acaso; interfere na precisão e, conseqüentemente, na reprodutibilidade do instrumento. O erro sistemático, ou viés, ocorre quando há, de forma repetida, diferença entre o valor medido e o verdadeiro, interferindo na validade do instrumento ${ }^{8}$.

Dessa forma, a qualidade das informações sobre a dieta será determinada pela precisão e pela validade do seu instrumento de registro, que deve ser avaliado especificamente para cada população em estudo ${ }^{9-11}$.

O presente trabalho avaliou a reprodutibilidade e a validade do QFCA utilizado no Estudo Latino-Americano sobre Câncer Oral e de Laringe, nos participantes do centro São Paulo. 


\section{Material e Método}

O Estudo Latino-Americano sobre Câncer Oral e de Laringe é um estudo caso-controle de base hospitalar, aprovado pela Comissão Nacional de Ética em Pesquisa (CONEP), desenvolvido com o objetivo de investigar os fatores de risco associados aos cânceres de cabeça e pescoço ${ }^{12}$. Foi conduzido em cinco centros no Brasil, um na Argentina (Buenos Aires) e um em Cuba (Havana), sob coordenação da Agência Internacional de Pesquisas em Câncer da Organização Mundial da Saúde (IARC/OMS).

No centro São Paulo, os 502 pacientes casos e os 442 controles eram procedentes de sete hospitais públicos. Os indivíduos foram questionados a respeito de sua história de exposição a diferentes fatores de risco para câncer oral, incluindo tabagismo, consumo de álcool, fatores ambientais e hábitos alimentares. As entrevistas foram realizadas entre novembro de 1998 e março de 2001. As informações sobre dieta foram coletadas utilizando-se um questionário de freqüência de consumo alimentar quantitativo composto por 26 alimentos (QFCA1): leite; iogurte; manteiga; pão; macarrão e arroz; pratos à base de milho; mandioca; farinha de mandioca; carne de boi; carne de porco; frango; peixe; embutidos; ovo; queijo; batata; vegetais crus; brócolis, repolho, couve de Bruxelas; cenoura; tomate; grãos; suco de frutas; maça e pêra; frutas cítricas; banana; bolo e doces.

\section{Definição da amostra}

Para a avaliação da reprodutibilidade e validade do QFCA do Estudo LatinoAmericano inicialmente foi selecionada uma amostra sistemática de 99 indivíduos, representando $10 \%$ dos indivíduos incluídos no centro São Paulo. Considerando a alta letalidade dos tumores orais e de laringe $^{13}$ e a possível modificação do padrão alimentar em função do diagnóstico de câncer, a seleção foi restrita aos indivíduos controles. Foram necessários três sorteios para compor o número estabele- cido da amostra, já que a ausência de telefone de contato no questionário original levava à exclusão do indivíduo sorteado.

Foram excluídos da amostra os indivíduos que não responderam à entrevista após três tentativas de contato, realizadas em dias alternados. Assim, da amostra inicial, 64 indivíduos foram excluídos, reduzindo-se a amostra final a 35 indivíduos. Os motivos de exclusão foram: três indivíduos por recusa em participar, quatro por mudança de residência, seis por ausência ou impossibilidade de atender, nove por falecimento e 42 pelo fato do número de telefone estar registrado incorretamente, desligado ou por ninguém atender.

Segundo Willett ${ }^{14}$ e Cade et al. ${ }^{15}$, a amostra para estudo de validade deve conter entre 50 e 100 indivíduos. Esta, porém, é uma recomendação. Há na literatura estudos recentes, publicados com um número variável de indivíduos, a partir de 20 sujeitos $^{16}$.

\section{Entrevista telefônica}

Os indivíduos selecionados foram contatados por telefone e entrevistados seguindo um roteiro de entrevista previamente elaborado. Antes da entrevista foram explicadas, para cada indivíduo, as condições de participação e foi solicitado seu consentimento. Os contatos telefônicos foram realizados de segunda-feira a domingo, no período de julho de 2003 a abril de 2004

$\mathrm{Na}$ entrevista telefônica os indivíduos responderam questões de ordem pessoal (idade e data de nascimento), dados antropométricos auto-referidos (peso e altura), o questionário de freqüência de consumo alimentar idêntico ao original do Estudo Latino-Americano (QFCA2) e um recordatório de 24 horas (R24h).

\section{Análise estatística}

Para análise, os 26 alimentos do QFCA foram reunidos em 15 grupos, de acordo com as características físicas e o valor nutricional dos alimentos: laticínios, man- 
teiga, pão, massas, tubérculos, carne de boi, carne de porco, frango, peixe, embutidos, ovo, hortaliças, leguminosas, frutas e doces. Bebidas alcoólicas, refrigerante, café e chá foram desconsiderados na análise.

Os dados obtidos em ambos os QFCA (QFCA1 e QFCA2) e no R24h foram tabulados em gramas de alimentos por dia utilizando o programa Virtual Nutri ${ }^{17}$.

A reprodutibilidade do questionário foi avaliada pela comparação, por grupo de alimento, do consumo descrito pelos indivíduos no QFCA1 e no QFCA2; a validade foi avaliada pela comparação do consumo descrito no R24h e no QFCA2.

O teste de Wilcoxon foi empregado para verificar a existência de diferenças no consumo registrado pelos três questionários; o coeficiente de correlação de Spearman, para verificar as correlações lineares no consumo e o coeficiente de correlação intraclasse (ICC), para estimar a variação intrapessoal no consumo.

Os indivíduos também foram classificados de acordo com o consumo, com base no R24h, em três categorias. A concordância no consumo foi verificada pelo percentual de indivíduos classificados em um mesmo tercil, e a discordância pelo percentual de indivíduos classificados nos tercis opostos (primeiro e terceiro tercis). Para a análise, a classificação em tercis foi comparada entre o QFCA1 e o QFCA2, e entre o R24h e o QFCA2, utilizando-se o teste Kappa.

Para as análises estatísticas foi utilizado o software SPSS, considerando-se um nível de significância de $5 \%$.

\section{Resultados}

Dos 35 indivíduos que responderam à entrevista completa, 28 eram do sexo masculino $(80 \%)$ e sete $(20 \%)$ eram do sexo feminino, com idade entre 37 e 81 anos (média de 59 anos). O tempo decorrido entre as duas entrevistas (no hospital e por telefone) variou de um ano e meio a quatro anos e meio (tempo médio de dois anos e oito meses).
A representação da amostra por grupo etário e por sexo seguiu o observado no Estudo Latino-Americano. Como divulgado pela International Agency for Research on Cancer $^{18}$, a razão de câncer oral entre homens e mulheres, no Brasil, é de 5:1. No Estudo Latino-Americano também foi encontrada esta razão e, como a amostragem foi aleatória, isso se refletiu na amostra final deste estudo.

A Tabela 1 apresenta a análise da reprodutibilidade. Comparando-se os valores de consumo de indivíduo a indivíduo através do teste de Wilcoxon, foram encontradas poucas diferenças significativas, demonstrando que o consumo descrito não se diferenciou entre os questionários. O consumo relatado no QFCA2 foi em geral maior que no QFCA1, com diferença estatisticamente significante $(p<0,05)$ apenas para os grupos de massas, de embutidos e de leguminosas, embora a diferença seja pequena quando consideradas as médias de consumo desses grupos. Observando os resultados do ICC foram constatadas baixas correlações para os grupos de peixe e frango, e razoáveis correlações (em torno de 0,2 ) para massas, tubérculos, carnes de boi e carne de porco. Os demais grupos apresentaram correlações acima de 0,32 .

$\mathrm{Na}$ análise da validade, quando comparado o consumo entre o R24h e o QFCA2 (teste de Wilcoxon), o consumo descrito no QFCA2 foi em geral maior que no R24h, com diferenças estatisticamente significativas para os grupos de manteiga, tubérculos, frango, hortaliças, leguminosas e frutas. A análise das diferenças observadas indicou que o QFCA2 subestimou o consumo do grupo de manteiga e superestimou o consumo dos demais grupos de alimentos (Tabela 2). O coeficiente de correlação de Spearman apresentou valores entre 0,36 e 0,71, com exceção dos grupos de carne de boi, carne de porco, peixe, embutidos e frutas, que se mostram inferiores a 0,32. As correlações foram estatisticamente significativas para dez dos 15 grupos de alimentos analisados. 
Tabela 1 - Médias de consumo, por grupo de alimentos, obtidas nos dois questionários de freqüência de consumo alimentar (QFCA1 e QFCA2), teste de Wilcoxon e coeficiente de correlação intraclasse.

Table 1 - Average of consumption by food group obtained by the food frequency questionnaires (FFQ1 and FFQ2), the Wilcoxon test and intraclass correlation coefficient.

\begin{tabular}{lcccc}
\hline Grupo & \multicolumn{3}{c}{ Consumo (g/dia) } & ICC \\
\cline { 2 - 4 } & Média QFCA1 & Média QFCA2 & Wilcoxon & \\
\hline Laticínios & 172,06 & 184,23 & 0,642 & 0,416 \\
Manteiga & 5,03 & 4,94 & 0,908 & 0,657 \\
Pão & 65,29 & 71,23 & 0,443 & 0,533 \\
Massas & 224,77 & 185,77 & $0,034^{*}$ & 0,224 \\
Tubérculos & 41,94 & 67,11 & 0,060 & 0,201 \\
Carne de boi & 59,03 & 49,63 & 0,383 & 0,244 \\
Carne de porco & 4,11 & 4,46 & 0,839 & 0,169 \\
Frango & 59,57 & 61,54 & 0,888 & 0,050 \\
Peixe & 16,40 & 11,57 & 0,364 & 0,063 \\
Embutidos & 5,54 & 11,29 & $0,050^{*}$ & 0,446 \\
Ovo & 13,83 & 16,83 & 0,356 & 0,424 \\
Hortaliças & 143,97 & 173,37 & 0,077 & 0,383 \\
Leguminosas & 176,46 & 138,49 & $0,031^{*}$ & 0,492 \\
Frutas & 345,54 & 387,94 & 0,326 & 0,318 \\
Doces & 13,03 & 16,20 & 0,599 & 0,421 \\
\hline
\end{tabular}

*Valor estatisticamente significante $(p<0,05)$ / Statistically significant value $(p<0.05)$

Tabela 2 - Médias de consumo, por grupo de alimentos, obtidas no recordatório de 24 horas (R24h) e no segundo questionário de freqüência de consumo alimentar (QFCA2), teste de Wilcoxon e coeficiente de Spearman.

Table 2 - Average of consumption by food group obtained by the 24-hour recall (R24h) and the second food frequency questionnaire (FFQ2), the Wilcoxon test and Spearman coefficient.

\begin{tabular}{lcccc}
\hline Grupo & \multicolumn{3}{c}{ Consumo (g/dia) } & \multirow{2}{*}{ Spearman } \\
\cline { 2 - 4 } & Média QFCA2 & Média R24h & Wilcoxon & \\
\hline Laticínios & 184,23 & 224,29 & 0,751 & $0,71^{*}$ \\
Manteiga & 4,94 & 13,91 & $0,008^{*}$ & $0,61^{*}$ \\
Pão & 71,23 & 65,74 & 0,333 & $0,57^{*}$ \\
Massas & 185,77 & 233,20 & 0,108 & $0,60^{*}$ \\
Tubérculos & 67,11 & 32,97 & $0,002^{*}$ & $0,46^{*}$ \\
Carne de boi & 49,63 & 77,20 & 0,221 & 0,05 \\
Carne de porco & 4,46 & 4,66 & 0,718 & 0,08 \\
Frango & 61,54 & 42,29 & $0,035^{*}$ & $0,37^{*}$ \\
Peixe & 11,57 & 15,09 & 0,680 & 0,32 \\
Embutidos & 11,29 & 7,14 & 0,240 & 0,19 \\
Ovo & 16,83 & 13,69 & 0,282 & $0,42^{*}$ \\
Hortaliças & 173,37 & 109,29 & $0,002^{*}$ & $0,39^{*}$ \\
Leguminosas & 138,49 & 105,97 & $0,032^{*}$ & $0,40^{*}$ \\
Frutas & 387,94 & 179,06 & $0,001^{*}$ & 0,22 \\
Doces & 16,20 & 35,97 & 0,278 & $0,36^{*}$ \\
\hline
\end{tabular}

*Valor estatisticamente significante $(p<0,05) /$ Statistically significant value $(p<0.05)$ 
Tabela 3 - Concordância da classificação dos indivíduos no mesmo tercil de consumo e em tercis opostos e teste Kappa entre os dois questionários de freqüência de consumo alimentar (QFCA1 e QFCA2) e entre o recordatório de 24 horas (R24h) e o segundo questionário (QFCA2).

Table 3 - Agreement of individuals' classification in the same tertile of food consumption and in discordant tertiles, and Kappa test between the two food frequency questionnaires (FFQ1 and FFQ2) and between the 24-hour recall (R24h) and the second food frequency questionnaire (FFQ2).

\begin{tabular}{|c|c|c|c|c|c|c|}
\hline \multirow[t]{2}{*}{ Grupo } & \multicolumn{3}{|c|}{$Q F C A 1 \times Q F C A 2$} & \multicolumn{3}{|c|}{$Q F C A 2 \times R 24 h$} \\
\hline & $\begin{array}{c}\text { Concordância } \\
\text { (\%) }\end{array}$ & $\begin{array}{c}\text { Tercis } \\
\text { Opostos (\%) }\end{array}$ & Kappa & $\begin{array}{c}\text { Concordância } \\
\text { (\%) }\end{array}$ & $\begin{array}{c}\text { Tercis } \\
\text { Opostos (\%) }\end{array}$ & Kappa \\
\hline Laticínios & 68,57 & 8,57 & $0,495^{*}$ & 68,57 & 5,71 & $0,526^{*}$ \\
\hline Manteiga & 71,43 & 0,00 & $0,373^{*}$ & 54,29 & 0,00 & $0,332^{*}$ \\
\hline Pão & 68,57 & 8,57 & $0,418^{*}$ & 71,43 & 8,57 & $0,493^{*}$ \\
\hline Massas & 48,57 & 5,71 & 0,125 & 57,14 & 5,71 & $0,352^{*}$ \\
\hline Tubérculos & 40,00 & 25,71 & 0,125 & 42,86 & 22,86 & $0,192^{*}$ \\
\hline Carne de boi & 65,71 & 0,00 & $0,216^{*}$ & 42,86 & 5,71 & 0,068 \\
\hline Carne de porco & 68,57 & 31,43 & $-0,027$ & 74,28 & 25,72 & 0,065 \\
\hline Frango & 65,71 & 11,43 & 0,173 & 42,86 & 42,86 & 0,146 \\
\hline Peixe & 48,57 & 51,43 & $-0,006$ & 68,57 & 31,43 & 0,216 \\
\hline Embutidos & 57,14 & 42,86 & 0,138 & 54,28 & 45,72 & 0,067 \\
\hline Ovo & 71,43 & 28,57 & $0,366^{*}$ & 54,28 & 45,72 & $0,231^{*}$ \\
\hline Hortaliças & 51,43 & 0,00 & $* *$ & 37,14 & 14,29 & 0,070 \\
\hline Leguminosas & 80,00 & 5,71 & $0,532^{*}$ & 45,71 & 14,29 & $0,198^{*}$ \\
\hline Frutas & 54,29 & 2,86 & $* *$ & 31,43 & 25,71 & $* *$ \\
\hline Doces & 51,43 & 48,57 & 0,023 & 65,71 & 34,29 & 0,307 \\
\hline
\end{tabular}

*Valor estatisticamente significante $(\mathrm{p}<0,05)$ / Statistically significant value $(p<0.05)$

**Valores não calculados, pois as categorias de resposta, de acordo com a classificação em tercis, não foram as mesmas nos questionários avaliados (condição necessária para o cálculo do teste Kappa) / Values not calculated, because the answer categories according to the classification in tertiles were not the same in the questionnaires (necessary condition for the Kappa test calculation).

O grau de concordância de classificação de indivíduos no mesmo tercil de consumo variou de $40 \%$ para tubérculos a $80 \%$ para leguminosas, entre os dois questionários de freqüência; e de $31 \%$ para o grupo de frutas a $74 \%$ para o grupo de carne de porco, entre o R24h e o QFCA2. O Kappa foi estatisticamente significante para os grupos de laticínios, manteiga, pão, carne de boi, ovo e leguminosas, quando comparados os dois QFCA, apontando concordância no consumo desses grupos. A concordância foi evidenciada também para os grupos de laticínios, manteiga, pão, ovo e leguminosas, além de massas e tubérculos, quando comparados o R24h e o QFCA2 (Tabela 3).

\section{Discussão}

Quando o Estudo Latino-Americano foi idealizado não foram previstos estudos complementares de reprodutibilidade e validade dos questionários que o compõem, inclusive o de hábitos alimentares. No decorrer do Estudo Latino-Americano, sentiu-se a necessidade da conhecer a acurácia e precisão do instrumento QFCA, especialmente em virtude das associações significativas observadas entre dieta e câncer oral ${ }^{19}$. Dessa forma, poderíamos avaliar com maior propriedade os resultados encontrados. Optou-se por utilizar os coeficientes de reprodutibilidade e validade, pois a oportunidade de discutir os resultados do Estudo Latino-Americano à luz destes resultados é particularmente valiosa, mesmo se tendo ciência dos possíveis vieses que possam ter sido inseridos nestas estimativas.

Diversos fatores podem afetar a reprodutibilidade dos instrumentos de registro do consumo alimentar, podendo ter influenciado os resultados desse estudo. Entre 
os possíveis fatores está o tempo decorrido entre as duas medições.

O efeito do tempo pode impactar em pelo menos duas importantes formas na medida da dieta habitual. Uma delas é obscurecer a memória da dieta passada, pois os estudos mostram que a recordação é influenciada pela dieta presente ${ }^{20}$. Outra é conseqüência de um intervalo de tempo muito grande podendo resultar em mudança nos hábitos alimentares, o que diminui a correlação entre os questionários e aumenta as diferenças entre as médias de consumo dos alimentos ${ }^{11,21}$. No entanto, não houve grandes modificações ambientais e ecológicas no intervalo de tempo decorrido entre o Estudo Latino-Americano e o presente estudo que tenham tido impacto no abastecimento e no acesso aos itens alimentares. Destaca-se ainda que a dieta na faixa social da população estudada é monótona e mantém um padrão relativamente constante.

Por outro lado, a reprodutibilidade das informações também demonstra uma variabilidade dependente da freqüência de consumo do alimento, sendo que os alimentos mais consumidos regularmente têm menor variação do que aqueles menos consumidos ${ }^{22}$. Consistente com esta assertiva, os piores resultados obtidos neste estudo foram observados nos alimentos que têm menor relato de ingestão, como carne de porco, peixe e embutidos.

Nos estudos de validade, Freedman et al. ${ }^{23}$ comentam que correlações de pequena magnitude podem ser resultado de relato com viés, ausência de padrão ouro e utilização de escala de medida diferencial no questionário de freqüência e no recordatório de 24 horas. Deve ser considerada mais uma vez a dificuldade dos indivíduos em lembrar os alimentos consumidos e a dificuldade de percepção e quantificação do tamanho das porções.

Outros aspectos que exercem influência sobre os relatos de consumo alimentar são o estado nutricional e a presença de doenças. Indivíduos com baixo peso tendem a superestimar a ingestão de gordu- ras; indivíduos obesos, ao contrário, tendem a subestimá-la ${ }^{22}$. Da mesma forma, a existência de certas doenças pode determinar um viés de memória.

Quanto à inexistência de um padrão ouro para validar a medida da dieta feita pelo QFCA, tem havido muito debate na comunidade científica, sendo unânime a opinião sobre a necessidade de buscarmos marcadores realmente independentes da dieta, como medidas bioquímicas da ingestão, pois os estudos têm demonstrado que o pressuposto teórico de independência de erros não é atendido ao usarmos os métodos de recordação ou registro da dieta como medidas de comparação ${ }^{24,25}$.

O método recordatório de 24 horas tem como característica elevada variabilidade intrapessoal, decorrente da natureza aleatória da dieta, que muda de um dia para outro e em função da sazonalidade. Assim, busca-se aplicar medidas repetidas deste método para possibilitar tanto a remoção do erro aleatório fruto da variância intrapessoal, quanto dos efeitos da sazonalidade. A aplicação única do R24h terá como efeito uma distribuição inflada do consumo de nutrientes, que atenuará as medidas de correlação. Assim sendo, as medidas obtidas no estudo poderão estar subestimadas em função deste efeito. Entretanto, as correlações entre as estimativas da dieta pelo QFCA e por métodos de referência freqüentemente se situam entre $0,3 \mathrm{e}$ 0,7 , o que sugere substancial erro ${ }^{26}$. Em nosso estudo, somente as correlações entre carne de boi, carne de porco, embutidos e frutas situaram-se abaixo de 0,3 . Verificou-se que a carne de boi, carne de porco e embutidos têm baixo consumo; para frutas, parece haver uma superestimação pelo QFCA. O mais preocupante é o valor obtido para a carne de boi, cuja baixa validade desacredita quaisquer associações decorrentes do consumo deste alimento.

Fraser e Stram ${ }^{26}$ mostraram ainda que o viés presente quando estimativas brutas dos dados do QFCA são utilizadas em uma regressão multivariada para estimar o efei- 
to da dieta na doença, é eliminado por uma regressão de calibração. Para obtenção do coeficiente de calibração, os autores postulam que é necessária apenas uma medida de referência.

Outro ponto a ressaltar é que nos estudos epidemiológicos analíticos, mais que estimar corretamente o consumo alimentar, busca-se classificar os indivíduos em gradientes de consumo para averiguar possíveis associações com o efeito ou doença investigada. A classificação dos indivíduos em tercis, utilizada no estudo, demonstrou baixo percentual de classificação em tercis opostos, em especial nos grupos de alimentos com maior consumo na população estudada. Para a validade, Slater et al. ${ }^{27}$ mostraram uma proporção muito similar de indivíduos classificados num mesmo tercil de consumo (33\%) e em tercis opostos (5\%).

Há que se enfatizar que o QFCA teve sua reprodutibilidade e validade avaliadas em função do Estudo Latino-Americano para investigação dos fatores de risco associados ao câncer oral. Foi opção metodológica não estudar os nutrientes e sim os alimentos, grupos de alimentos e padrão alimentar. Em face da complexidade da dieta, e especialmente em virtude da elevada correlação entre os nutrientes, estudiosos como Willett ${ }^{14}$ recomendam diversas abordagens para a avaliação dos efeitos da exposição aos fatores dietéticos. Ressalta-se que a abordagem dos efeitos dos grupa- mentos e padrões de consumo alimentar tem recebido maior atenção na atualidade, pois, entre outros fatores, os achados podem ser facilmente transformados em recomendações compreensíveis para a população. Além disso, há um menor número de estudos de validade e reprodutibilidade com alimentos, e, assim, este estudo espera contribuir tanto para a melhor interpretação dos resultados do Estudo Latino-Americano, o maior realizado em nosso meio até a presente data, como para o debate científico nesta área.

A existência de poucos estudos publicados de reprodutibilidade e validade com alimentos - apenas um no Brasil - inviabilizou a comparação dos resultados obtidos com estudos nacionais. No único estudo publicado $^{10}$ verificou-se a reprodutibilidade do questionário quanto ao consumo de frutas frescas e ao consumo de vegetais, sendo que o tempo decorrido entre as aplicações foi de apenas duas semanas.

Em nosso estudo foram obtidos coeficientes de reprodutibilidade e validade semelhantes aos da literatura para a maioria dos grupos de alimentos estudados ${ }^{28,29}$. Os resultados indicam que o questionário de freqüência empregado no Estudo LatinoAmericano sobre Câncer Oral e de Laringe para estimar o consumo de alimentos, a exceção de carne de boi e carne de porco, tem boa reprodutibilidade e razoável validade, por superestimar moderadamente o consumo de alguns grupos de alimentos.

\section{Referências}

1. Willet WC. Diet and nutrition. In: Schottenfeld D, Fraumeni Jr JF (ed.). Cancer epidemiology and prevention. New York: Oxford University Press; 1996. p. 438-61.

2. World Health Organization. World cancer report. Lyon: IARC Press; 2003.

3. Instituto Nacional do Câncer [on line]. Disponível em http://www.inca.gov.br Acessado em 1 de junho de 2006.
4. Jimenéz LG, Moreno JMM. Cuestionário de frecuencia de consumo alimentario. In: Majem LS, Bartrina JA, Verdú JM (ed.). Nutrición y salud pública: métodos, bases científicas y aplicaciones. Barcelona: Masson; 1995. p. 120-25.

5. Salvo VLMA. Construção de um questionário de freqüência de consumo de alimentos para adultos com excesso de peso: estudo de reprodutibilidade e validade indireta [dissertação de mestrado]. São Paulo: Escola Paulista de Medicina da Universidade Federal de São Paulo; 2000. 
6. López JL. Validez de la evaluación de la ingesta dietética. In: Majem LS, Bartrina JA, Verdú JM (ed.). Nutrición y salud pública: métodos, bases científicas y aplicaciones. Barcelona: Masson; 1995. p. 132-36.

7. Fisberg RM, Slater B, Marchioni DML, Martini LA. Inquéritos alimentares: métodos e bases científicas. Barueri: Manole; 2005.

8. Torrell JMR. Reproductibilidade de las encuestas alimentarias. In: Majem LS, Bartrina JA, Verdú JM (ed.). Nutrición y salud pública: métodos, bases científicas y aplicaciones. Barcelona: Masson; 1995. p. 137-40.

9. Rodríguez MM, Méndez H, Torún B, Schroeder D, Stein $\mathrm{AD}$. Validation of a semi-quantitative food-frequency questionnaire for use among adults in Guatemala. Public Health Nutr 2002; 5(5): 691-8.

10. Chor D, Faerstein E, Alves MGM, Lopes CS. How reproducible is self-reported information on exposure to smoking, drinking, and dietary patterns? Evidence among Brazilian adults in the Pró-Saúde Study. São Paulo Med J 2003; 121(2): 63-6.

11. Shu XO, Yang G, Liu D, Kushi L, Wen W, Gao YT, Zheng W. Validity and reproducibility of a food frequency questionnaire used in the Shanghai Women's Health Study. Eur J Clin Nutr 2004; 58: 17-23.

12. Boffetta P, Brennan P, Herrero R. International study of environment, viruses and cancer of the oral cavity and the larynx. Study Protocol. Lyon: IARC; 1998 (mimeo).

13. Departamento de Informação e Informática do SUS. Informações de saúde: estatísticas vitais [on line]. Disponível em http:/ / www.datasus.gov.br Acessado em 21 de junho de 2006.

14. Willett W C. Nutritional epidemiology. New York: Oxford University Press; 1998.

15. Cade J, Thompson R, Burley V, Warm D. Development, validation and utilization of food-frequency questionnaires: a review. Public Health Nutr 2002; 5(4): 567-87.

16. Nath SD, Huffman FG. Validation of a semiquantitative food frequency questionnaire to assess energy and macronutrient intakes of Cuban Americans. Int J Food Sci Nutr 2005; 56(5): 309-14.

17. Philippi ST, Szartarc SC, Latterza AR. Virtual nutri (software), versão 1.0 para Windows. São Paulo; 1996: Departamento de Nutrição, Faculdade de Saúde Pública, Universidade de São Paulo.

18. International Agency for Research on Cancer. Globocan 2002 database: summary table by population [on line]. Disponível em http://www-dep.iarc.fr Acessado em 1 de junho de 2006.
19. Marchioni DML et al. Fatores dietéticos e câncer oral: estudo caso-controle na região metropolitana de São Paulo. Cad Saúde Pública; 2006 (no prelo).

20. Krall EA, Dwyer JT, Coleman KA. Factors influencing accuracy of dietary recall. Nutr Res 1988; 8: 829-41.

21. Pereira RA, Koifman S. Uso do questionário de freqüência na avaliação do consumo pregresso. Rev Saúde Pública 1999; 33(6): 610-21.

22. Slater B. Desenvolvimento e validação de um questionário semi-quantitativo de freqüência alimentar para adolescentes [tese de doutorado]. São Paulo: Faculdade de Saúde Pública da Universidade de São Paulo; 2001.

23. Freedman LS, Carroll RJ, Wax Y. Estimating the relation between dietary intake obtained from a food frequency questionnaire and true average intake. Am J Epidemiol 1991; 134: 310-20.

24. Day NE, Ferrari P. Some methodological issues in nutritional epidemiology. IARC Sci Publ 2002; 156: 5-10.

25. Bingham SA, Gill C, Welch A, Cassidy A, Runswick SA, Oakes $\mathrm{S}$ et al. Validation of dietary assessment methods in the UK arm of EPIC using weighed records, and 24-hour urinary nitrogen and potassium and serum vitamin $C$ and carotenoids as biomarkers. Int J Epidemiol 1997; 26(S1): S137-51.

26. Fraser GE, Stram DO. Regression calibration in studies with correlated variables measured with error. $A m J$ Epidemiol 2001; 154 (9): 836-44.

27. Slater B, Philippi ST, Fisberg RM, Latorre MRDO. Validation of a semi-quantitative adolescent food frequency questionnaire applied at a public school in São Paulo, Brazil. Eur J Clin Nutr 2003; 57: 629-35.

28. Mannisto S, Virtanen M, Mikkonen T, Pietinen P. Reproducibility and validity of a food frequency questionnaire in a case-control study on breast cancer. $J$ Clin Epidemiol 1996; 49(4): 401-9.

29. Marks G C, Hughes M C, van der Pols J C. Relative validity of food intake estimates using a food frequency questionnaire is associated with sex, age, and other personal characteristics. J Nutr 2006; 136(2): 459-65.

recebido em: 01/12/05

versão final reapresentada em: 29/08/06 aprovado em: 29/08/06 